that can potentially be used on the neonatal units and postnatal wards.

Aim To develop neonatal track and trigger observation chart in order to enable early identification of neonates in need of urgent medical assessment and intervention.

Methods A core group involving local paediatricians, neonatal nurses and midwifery sister was established to lead the project. The group contacted various neonatal units in different newborn networks in England seeking information if early warning scores or track \& trigger system was being developed or already well established. Literature search was carried out to identify studies related to newborn early warning system scores.

Results One relevant published study was retrieved from Medline search (Roland 2010). None of the neonatal units contacted had an established early warning neonatal scoring system. Group developed newborn observation chart for "At Risk" and "High Risk" Infants. It was based on neurophysiological parameters, intervention criteria and staff concerns. A decision tree was devised based on trigger scores.

Conclusions Prospectively evaluation of Burton neonatal track and trigger observation chart is required to ascertain its efficacy. If found to be reliable and valid, it will facilitate observation of neonates deemed to be at risk and prompt an early review in triggered neonates.

\section{CARDIAC INVOLVEMENT IN YOUNG INFANTS WITH SEPSIS-LIKE ILLNESS IS NOT ASSOCIATED WITH ENTEROVIRUS INFECTION}

doi:10.1136/archdischild-2012-302724.1569

'EP De Jong, 'LHPM Filippini, ${ }^{2}$ EPM Van Elzakker, ${ }^{3} F J$ Walther, ${ }^{1} F$ Brus. ' Juliana Children's Hospital; ${ }^{2}$ Medical Microbiology, HAGA Hospital, The Hague; ${ }^{3}$ Paediatrics, Division of Neonatology, Leiden University Medical Center, Leiden, The Netherlands

Introduction Enterovirus (EV) infection is common in young infants, amongst those admitted to intensive care units acute myocarditis has been reported. We questioned if myocardial involvement exists in less seriously ill children with EV infection.

Methods From august 2011 onward we included children under 90 days of age, who were admitted to our hospital for sepsis-like illness. During admission serum concentrations of Troponine-I, CK, $\mathrm{CK}-\mathrm{MB}, \mathrm{BNP}$ and NT-Pro-BNP were determined and an electrocardiogram and echocardiography were performed. Differences between children with and without EV infection were studied.

Results We present results of the first 28 patients included, 14 were EV positive. Basic patient characteristics were similar between $\mathrm{EV}$ positive and negative infants. In 17/28 infants cardiac enzymes could be determined. CK was normal in all, CK-MB was elevated in 11 infants, Troponin-I in 2, BNP in 14 and NT-Pro-BNP in all but one. There was no difference in cardiac enzyme concentration between the two groups.

Electrocardiograms showed signs of ischemic heart disease in two infants that disappeared at follow-up four weeks later. One was EV positive and one negative. In both cases not enough material was collected to evaluate cardiac enzymes.

None of the children showed signs of cardiac dysfunction at echocardiography.

Conclusion Regarding signs of cardiac involvement no differences were found between EV positive and negative infants with sepsislike illness. Both groups showed elevation of cardiac enzymes. Cardiac involvement seems to be subtle, only 2 infants showed transient ischemia on ECG whereas none showed myocardial dysfunction on conventional echocardiography.

\section{PROGNOSTIC PARAMETERS AND OUTCOME OF INVASIVE MENINGOCOCCAL DISEASE IN CHILDREN}

doi:10.1136/archdischild-2012-302724.1570
'V Zilinskaite, ${ }^{2} \mathrm{~L}$ Peciuliene, ${ }^{2} \mathrm{~L}$ Sakalauskaite. ${ }^{1} \mathrm{PICU}$, Vilnius University Clinic of Children's Diseases, ${ }^{2} P I C U$, Children's Hospital, Affiliate of Vilnius University Hospital Santariskiu Klinikos, Vilnius, Lithuania

The Aim of this study was to evaluate retrospectively the clinical prognostic parameters of children with diagnosed invasive meningococcal disease (IMD) during their admission to the emergency department and compare them to clinical diagnosis and outcome.

Methods We retrospectively evaluated 75 patients admitted to the PICU of Vilnius University Children's Hospital during 2009-2010 years. IMD with meningitis was diagnosed in 32 patients (McM group), meningococcemia and septic shock without meningitis in 43 patients (Mc group). 6 patients died (8\%), 5 in Mc group (11.6\%), and 1 in McM group (3.1\%). 6 bad prognosis parameters (BPP) during admission were evaluated:

1. short duration of illness before admission (less than 24 hours),

2. widespread haemorrhagic rash,

3. signs of septic shock,

4. alert state of consciousness,

5. blood leukocyte count $<10 \times 109 / \mathrm{L}$

6. blood platelet count $<100 \times \sigma 109 / \mathrm{L}$

Results All 6 BPP were established in $6 \mathrm{Mc}$ group patients, 5 of them died, all have duration of illness less than 12 hours. 4 or more BPP was in 16 (37.2\%) Mc group patients, and in $6(18.7 \%) \mathrm{McM}$ ( $p>0.05) .3$ or less BPP was equal in both groups. More than 2 BPP was established statistically significant more often in Mc patients group $-60.5 \%$ and $34.4 \%$ ( $\mathrm{p}=0.036)$.

Conclusions An early identification child with meningococcal sepsis without meningitis is important to start the appropriative treatment as soon as possible. 2 or more BPP during the first assessment in the emergency department can be predictive values of the life threatening process of meningococcal infection.

\section{RISKS FACTORS FOR METHICILLIN-RESISTANT STAPHYLOCOCCUS AUREUS (MRSA) ACQUISITION IN PEDIATRIC INTENSIVE CARE UNIT (PICU)}

doi:10.1136/archdischild-2012-302724.1571

MH Rathore. Department of Pediatrics, University of Florida, Jacksonville, FL, USA

Background MRSA is a significant problem in ICUs. Data on risks for acquiring MRSA while in PICU are minimal.

Methods Children < 19 years old admitted to PICU between 4/1/2008 \& 3/31/2011 had admission \& weekly MRSA nasal surveillance done. MRSA affected (colonized or infected) children were compared to unaffected.

Results There were 2861 admissions. Mean age 6.7 yrs (median 4.7); 1444 (50.5\%) male. 2632 (92\%) of 2861 either had a known history of MRSA or an admission surveillance test and were included in the analysis. 415 (15.8\%) were MRSA affected; 264 had known history of MRSA, 132 were MRSA+ on admission and 19 became affected while in PICU (18 colonized \& 1 infected). $14(77.8 \%)$ of 18 colonized were identified on weekly surveillance, $4(22.2 \%)$ had a positive non-surveillance culture.19 children who became MRSA affected were further analyzed. There was no significant difference in gender or ethnicity between the two groups MRSA affected were younger ( 3.68 vs 6.79 yrs, $p=0.03$ ). Mean Hospital length of stay (LOS) prior to PICU admission was longer in the MRSA affected group (2.3 vs 0.6 days, $p=0.04$ ). Systemic steroids $(\mathrm{p}=0.009)$, mechanical ventilation $(\mathrm{p}=0.001)$ and a central venous catheter (CVC) $(p=0.001)$ were all higher in the MRSA affected group; surgery \& antibiotic use were not. Mean LOS in the PICU was 4.3 days, Mean LOS in the PICU before becoming MRSA affected was 18 days. 